\title{
Erratum to: A higher rank Lefschetz formula
}

Anton Deitmar

\section{Erratum to: J. fixed point theory appl. 2 (2007), 1-40 DOI 10.1007/s11784-007-0028-3}

The proofs in [1] contain inaccuracies which we amend in this erratum. The main results of that paper remain untouched by these corrections.

\section{Smoothness of induced functions}

For Proposition 1.4.1 to be correct, one has to insist that the open set $C$ be regular in the sense that $C$ is the open interior of its closure. In this way the boundary of $C$ is also the boundary of the complement.

Furthermore, in order to apply Proposition 1.4.1 to the construction of the test function $f$ in Section 4.3, the condition $\partial F(C) \subset F(\partial C)$ needs to be satisfied. As it stands (page 35), this condition is not satisfied. One can, however, replace the set $N$ in the definition of the set $C$ on page 35 with an open subset $U$ of $N$ which has compact support. This suffices for the application and then the proof of the property $\partial F(C) \subset F(\partial C)$ runs as follows: Let $\left(k_{j}, n_{j}, m_{j}, a_{j}\right)$ be a sequence in $C$ such that $F\left(k_{j}, n_{j}, m_{j}, a_{j}\right)=$ $k_{j} n_{j} a_{j} m_{j} n_{j}^{-1} k_{j}^{-1}$ converges to some $g \in G$. As $K$ is compact, we can assume that $k_{j}$ converges to some $k \in K$. Replacing $g$ with $k^{-1} g k$ we reduce to $k=1$, so the sequence $n_{j} a_{j} m_{j} n_{j}^{-1}$ converges to $g$. As $n_{j}$ varies in a relatively compact set, we can assume that $n_{j}$ converges to some $n \in N$. We have

$$
n_{j} a_{j} m_{j} n_{j}^{-1}=\underbrace{a_{j}}_{\in A} \underbrace{m_{j}}_{\in M} \underbrace{n_{j}^{a_{j} m_{j}} n_{j}^{-1}}_{\in N} .
$$

As the product $A M N$ is direct, we conclude that $a_{j}$ and $m_{j}$ converge as well to some $a \in A$ and $m \in M$. We get $g=\operatorname{namn}^{-1}$ must be in $F(\partial C)$ as claimed.

The online version of the original article can be found under doi:10.1007/s11784-007-0028-3. 


\section{The trace formula}

The proof of the trace formula in Proposition 3.1.2 of [1] only works for functions in $L_{2 N}^{2}(G)$ which are positive, i.e., $\geq 0$ or linear combinations thereof. So the proposition needs to be replaced with the following.

Proposition. Suppose $N>\frac{\operatorname{dim} G}{2}$. Then the spectral side of the trace formula defines a continuous linear functional on $L_{2 N}^{1}(G)$. If $f$ lies in $L_{2 N}^{1,+}(G)$, then the trace formula holds for $f$.

The proof is the same as in [1], except for the fact that Lemma 3.1.1 is not invoked. This restriction of the trace formula has no influence on the final results of that paper, as it is easy to show that the space $C^{\mu, j}\left(A^{-}\right)$is spanned by its positive members and the Euler-Poincaré function $f_{\tau}$ can be written as a linear combination of positive, compactly supported functions since $f_{\tau}$ itself has compact support.

\section{The test function}

The construction of the function $\chi_{p}: A^{-} M \rightarrow[0,1]$ on page 33 of [1] needs correction. As constructed, this function is not $p$-times continuously differentiable as announced. We replace the construction with the following.

Let $\tilde{E} \subset \mathbb{C}^{n}$ be the compact set of all $z_{1}, \ldots, z_{n}$ with $\left|z_{1}\right|,\left|z_{2}\right|, \ldots,\left|z_{n}\right| \leq$ 1 , and let $E$ be the image of $\tilde{E}$ in $\mathbb{C} / \operatorname{Per}(n) \cong \mathbb{C}^{n} \cong \mathbb{R}^{2 n}$. We identify $\mathbb{C} / \operatorname{Per}(n)$ with $\mathbb{R}^{2 n}$ by means of the elementary symmetric polynomials which also give us an analytic map $\sigma: \mathbb{C}^{n} \rightarrow \mathbb{R}^{2 n}$. The map $\sigma$ is surjective, analytic, and has the property that its fibres are the $\operatorname{Per}(n)$-orbits. Being the image of of the compact set $\tilde{E}$ under a continuous map, the set $E \subset \mathbb{R}^{2 n}$ is compact. We also get a map from the set $\operatorname{Mat}_{m}(\mathbb{C})$ of all complex $n \times n$ matrices to $\mathbb{C}^{n} / \operatorname{Per}(n)$ sending a matrix to its eigenvalues. We thus get a map $\operatorname{Mat}_{n}(\mathbb{C}) \rightarrow \mathbb{R}^{2 n}$, which, by abuse of notation, we also denote by $\sigma$. Its coordinates are

$$
\sigma_{k}(m)=\operatorname{tr} \wedge^{k} m, \quad k=1, \ldots, n .
$$

Let $\ell(z)=\log \left(\max \left(1,\left|z_{1}\right|, \ldots,\left|z_{n}\right|\right)\right)$. Then $\ell(z)=0 \Leftrightarrow z \in \tilde{E}$ and $\ell$ descends to a continuous function of $\mathbb{C}^{n} / \operatorname{Per}(n) \cong \mathbb{R}^{2 n}$.

Lemma 3.1. Let $d$ denote the euclidean distance in $\mathbb{R}^{2 n}$. Let $N, p \in \mathbb{N}$ and let $E \subset \mathbb{R}^{N}$ be a compact set. Then there exists a function $d_{E, p}: \mathbb{R}^{N} \rightarrow[0, \infty)$, which is $(p-1)$-times continuously differentiable such that for every $x \in \mathbb{R}^{N}$ one has

$$
d_{E, p}(x) \geq d(x, E)^{p} \quad \text { and } \quad d_{E, p}(x)=0 \Leftrightarrow x \in E .
$$

Proof. Let $\varphi \in C_{0}^{\infty}\left(\mathbb{R}^{N}\right)$ have support equal to the closed ball $\bar{B}_{1}(0)$ of radius 1 around zero. Suppose that

$$
\varphi \geq 0 \text { and } \int_{\mathbb{R}^{N}} \varphi(x) d x=1 .
$$


For $r>0$ let $\varphi_{r}(x)=\frac{1}{r} \varphi\left(\frac{x}{r}\right)$. Then $\operatorname{supp}\left(\varphi_{r}\right)=\bar{B}_{r}(0)$ and $\int_{\mathbb{R}^{N}} \varphi_{r}(x) d x=1$. Let $D$ be the set of all $x \in \mathbb{R}^{N}$ with $d(x, E)>1$. Set $h_{0}(x)=\mathbf{1}_{D}(x) d(x, E)^{p+1}$ and let $d_{E, p}^{0}$ be the convolution product

$$
d_{E, p}^{0}=c_{0} h_{0} * \varphi_{1 / 4},
$$

where the constant $c_{0}>0$ is the smallest number such that $d_{E, p}^{0}(x) \geq$ $d(x, E)^{p}$ holds for every $x \in D$.

For $n=0,1,2 \ldots$ set

$$
B_{n}=\left\{x \in \mathbb{R}^{N}: \frac{1}{2^{n}}<d(x, E)<\frac{2}{2^{n}}\right\} .
$$

For $n=1,2,3, \ldots$ define $h_{n}(x)=\mathbf{1}_{B_{n-1} \cup B_{n} \cup B_{n+1}}(x) d(x, E)^{p}$ and set

$$
d_{E, p}^{n}=c_{n} h_{n} * \varphi_{2^{-(n+2)}},
$$

where the constant $c_{n}>0$ is the smallest number such that $d_{E, p}^{n} \geq d(x, E)^{p}$ holds for every $x \in B_{n}$. Set

$$
d_{E, p}=\sum_{j=0}^{\infty} d_{E, p}^{j} .
$$

The sum is locally finite outside $E$, so the function $d_{E, p}$ is smooth outside $E$. There is $C>0$ with $c_{n}<C$ for every $n \geq 0$.

For $n \geq 1$ one has $h_{n}(x) \leq 2^{(1-n) p}$, so the convergence is uniform on $\mathbb{R}^{N}$. Let $D$ be a differential operator with constant coefficients on $\mathbb{R}^{N}$ of degree $k$. Then

$$
\begin{aligned}
\left|D d_{E, p}^{n}\right| & =c_{n}\left|D\left(h_{n} * \varphi_{2^{-(n+2)}}\right)\right| \\
& =c_{n}\left|h_{n} *\left(D \varphi_{2^{-(n+2)}}\right)\right| \\
& \leq 2^{(n+2) k} c_{n}\left|h_{n} *(D \varphi)_{2^{-(n+2)}}\right| \\
& \leq \text { const } 2^{(n+2) k} 2^{(1-n) p} .
\end{aligned}
$$

We see that, as long as $k<p$, the sum $\sum_{n=0}^{\infty} D d_{E, p}^{n}$ converges uniformly, hence defines a continuous function. Integrating this function we get back $d_{E, p}$, which therefore is $(p-1)$-times continuously differentiable. The lemma is proven.

Back to the case when $N=2 n$ and $E \subset \mathbb{R}^{2 n}$ is the image of $\tilde{E}$.

Lemma 3.2. There exists $m \in \mathbb{N}$ such that for every $p \in \mathbb{N}$ there exists a map $d_{p}: \mathbb{R}^{2 n} \rightarrow[0, \infty)$, which is $(p-1)$-times continuously differentiable, such that

$$
d_{p}(x)=0 \Longleftrightarrow x \in E,
$$

and $d_{p}(x) \geq \ell(x)^{p m}$ holds for every $x \in \mathbb{R}^{2 n}$.

Proof. In order to employ the last lemma, we need to prove the existence of $m \in \mathbb{N}$ such that for $x \in \mathbb{R}^{2 n}$ one has $l(x)^{m} \leq C d(x, E)$ for some constant 
$C>0$. In terms of $z \in \mathbb{C}^{n}$ this amounts to the following. We write $\|z\|_{\infty}=$ $\max \left(\left|z_{1}\right|, \ldots,\left|z_{n}\right|\right)$. We claim that there exists $m \in \mathbb{N}$ and $C>0$ such that

$$
\left(\log \|z\|_{\infty}\right)^{m} \leq C d(\sigma(z), E) .
$$

As $\|z\|_{\infty} \rightarrow \infty$, it follows that $d(\sigma(z), E)$ is asymptotic to $\|\sigma(z)\|$, the euclidean norm. This means that the right-hand side grows faster than the left. Further, if $\alpha>1$, then the set of all $\sigma(z)$, where $\|z\|_{\infty} \geq \alpha$, is a closed set disjoint from the compact $E$, so that $d(\sigma(z), E)$ takes a minimum on that set. It follows that for every $\alpha>1$ there exists $C_{\alpha}>0$ such that the claimed estimate holds for every $z$ with $\|z\|_{\infty} \geq \alpha$. So all we have to show is that the estimate remains true with a fixed $C>0$ as $\|z\|_{\infty} \rightarrow 1$ from above. As $\|z\|_{\infty}$ tends to 1 , we can replace $\log \|z\|_{\infty}$ by $\|z\|_{\infty}-1$. We assume the claim is false. Then for every $j \in \mathbb{N}$ there exist $z_{j}, w_{j} \in \mathbb{C}^{n}$ with $\left\|w_{j}\right\|_{\infty} \leq 1<\left\|z_{j}\right\|_{\infty}$ such that

$$
\frac{\left\|\sigma\left(z_{j}\right)-\sigma\left(w_{j}\right)\right\|^{2}}{\left(\left\|z_{j}\right\|_{\infty}-1\right)^{2 j}}<\frac{1}{j}
$$

and $\left\|z_{j}\right\|_{\infty} \rightarrow 1$. The sequences $\left(z_{j}\right)$ and $\left(w_{j}\right)$ remain in a compact set, replacing them by subsequences if necessary, we can assume they converge, say $z_{j} \rightarrow z_{0}$ and $w_{j} \rightarrow w_{0}$. Write $z_{j}=\left(z_{j, 1}, \ldots, z_{j, n}\right)$. We are free to replace $z_{j}$ with any element of $\operatorname{Per}(n) z_{j}$, so we can assume that $\left|z_{j, 1}\right|=\left\|z_{j}\right\|_{\infty}$. Multiplying everything with a complex number of absolute value one, we can assume that $z_{1, j}=t_{j}>0$. Let $z_{j}^{\prime}=\left(z_{j, 2}, \ldots, z_{j, n}\right)$. We now split $z$ and $w$ into real and imaginary parts as $z^{\prime}=x+i y$ and $w=u+i v$. Then the function of real variables

$$
f\left(t, z^{\prime}, w\right)=f(t, x, y, u, v)=\|\sigma(t, x+i y)-\sigma(u+i v)\|^{2}
$$

is real analytic. We consider the power-series expansion around $t=1$ :

$$
f\left(t, z^{\prime}, w\right)=\sum_{j=0}^{\infty}(t-1)^{j} f_{j}\left(z^{\prime}, w\right) .
$$

We know that

$$
\left|f\left(t_{j}, z_{j}^{\prime}, w_{j}\right)\right| \leq\left|\frac{f\left(t_{j}, z_{j}^{\prime}, w_{j}\right)}{\left(t_{j}-1\right)^{2 j}}\right|<\frac{1}{j}
$$

for every large enough $j$. It follows that $f_{0}\left(z_{0}^{\prime}, w_{0}\right)=0$. We iterate this argument to get $f_{j}\left(z_{0}^{\prime}, w_{0}\right)=0$ for all $j$ which implies

$$
f\left(t, z_{0}^{\prime}, w_{0}\right)=0
$$

for all $t$. The latter, however, is absurd, as we know that $f\left(t, z_{0}^{\prime}, w_{0}\right)$ tends to infinity, as $t \rightarrow+\infty$. The lemma is proven.

Let $\eta: \mathbb{R} \rightarrow[0,1]$ be a smooth function such that

$$
\eta(x)=1 \text { if } x \leq \frac{1}{3} \quad \text { and } \quad \eta(x)=0 \text { if } x \geq \frac{2}{3} .
$$


Next let $\varphi \in C_{c}^{\infty}\left(\mathbb{R}^{2 n}\right)$ be such that $\varphi \geq 0$ and $\varphi(x)>0 \Leftrightarrow\|x\|<\frac{1}{3}$, as well as $\int_{\mathbb{R}^{2 n}} \varphi(x) d x=1$. Next let

$$
\varphi_{\tau}(x)=\frac{1}{\tau} \varphi\left(\frac{x}{\tau}\right)
$$

and define

$$
h_{\tau}^{p}(x)=\eta\left(\frac{d_{p}(x)}{\tau}\right)
$$

as well as

$$
g_{p}(\tau, x)=h_{\tau}^{p} * \varphi_{\tau}(x),
$$

where the star indicates the convolution product on $\mathbb{R}^{2 n}$. For $R>0$ let $U_{R}$ be the set of all $x \in \mathbb{R}^{2 n}$ with $d_{p}(x)<R$. Then $\operatorname{supp}\left(h_{\tau}^{p}\right)=\overline{U_{2 \tau / 3}}$ and $\operatorname{supp}\left(g_{p}(\tau, \cdot)\right)=\overline{U_{\tau}}$.

The convolution product makes $g_{p}(\tau, x)$ a smooth function in the $x$ variable. It is also a smooth function in the $\tau$-variable as all its factors are. Further, all partial $x$ - or $\tau$-derivatives are $(p-1)$-times continuously differentiable in the other argument as well, so finally, this function is $p$-times continuously differentiable as a function on $\mathbb{R}^{+} \times \mathbb{R}^{2 n}$. It satisfies $0 \leq g_{p} \leq 1$ and $g_{p}(\tau, x)=0$ if $d_{p}(x)>\tau$, as well as $g(\tau, E)=1$. Further, for every multiindex $\alpha \in \mathbb{N}_{0}^{2 n+1}$ there exists a constant $c_{\alpha}>0$ such that for a differential operator $D=\sum_{\alpha} a_{\alpha} \partial^{\alpha}$ of degree $\leq p$ on $\mathbb{R}^{+} \times \mathbb{R}^{2 n}$ with smooth coefficients $a_{\alpha}$, one gets

$$
|D g(\tau, x)| \leq \max _{d(y, x) \leq \tau / 3} \sum_{\alpha} c_{\alpha}\left|a_{\alpha}(\tau, y)\right|\left(\frac{1}{\tau}\right)^{|\alpha|} .
$$

Note that with $g_{p, \tau}(x)=g_{p}(\tau, x)$, the support of $g_{p, \tau}$ is compact for every $\tau>0$, contains $E$, and shrinks monotonically to $E$, as $\tau \rightarrow 0$.

We now apply this to $n=\operatorname{dim} \mathfrak{g}$. The adjoint representation then maps $M$ to $\mathrm{SL}(\mathfrak{g}) \cong \mathrm{SL}_{n}(\mathbb{C})$. We have $\sigma\left(M_{\text {ell }}\right) \subset E$. For $a m \in A^{-} M$ we have

$$
a m \in(A M)^{\sim} \Longleftrightarrow \ell(\sigma(m))<\frac{\log \left(\lambda_{\min }(a \mid \overline{\mathfrak{n}})\right)}{2} .
$$

Let $U$ be an open subset of a manifold $M$. For $k \geq 0$, let $C_{0}^{k}(M, U)$ denote the space of all functions $f$ on $U$ such that

- extended by zero outside $U$, the function $f$ is $k$-times continuously differentiable, and

- $f$ is smooth inside $U$.

For $k \leq K$ let $C_{0}^{k, K}(M, U)$ be the set of all functions in $C_{0}^{k}(M, U)$ such that

$$
\frac{1}{f} C_{0}^{K+j+1}(M, U) \subset C_{0}^{j}(M, U) \text { holds for every } 0 \leq j .
$$

We have the following examples.

- For $M=\mathbb{R}$ and $U=(0, \infty)$, the function $f(x)=x^{k+1}$ is in $C_{0}^{k, k}(M, U)$.

- For $M=\mathbb{R}^{N}$ and $U$ being the open unit ball, the function $f(x)=$ $\left(1-\|x\|^{2}\right)^{k+1}$ is in $C_{0}^{k, k}(M, U)$. 
- For $M=\mathbb{R}^{N}$ and $U=\left\{x \in \mathbb{R}^{N}: x_{1}, \ldots, x_{N}>0\right\}$, the function $f(x)=\left(x_{1} \cdots x_{n}\right)^{k+1}$ is in $C_{0}^{k, k}(M, U)$.

Lemma 3.3. There exists a function $\tau: A \rightarrow[0,1]$ such that

- $\tau$ is in $C_{0}^{m p, m p}\left(A, A^{-}\right)$;

- $0<\tau(a)<\left(\frac{\log \left(\lambda_{\min }(a \mid \overline{\mathfrak{n}})\right)}{2}\right)^{p m}$ if $a \in A^{-}$;

- for every $D \in U(\mathfrak{a})$ of degree $\leq m p$ the function $D \tau$ is bounded.

Proof. Let $\alpha_{1}, \ldots, \alpha_{r}$ be the simple roots of $(A, \bar{P})$, where $P$ is the parabolic $M A \bar{N}$ and $r=\operatorname{dim} A$. The group $A$ is isomorphic to its Lie algebra and the latter is mapped isomorphically to $\mathbb{R}^{r}$ by a map $\alpha$ with coordinates $\alpha_{j}$. In this map the cone $A^{-}$is mapped to the positive cone $C^{+}$of all $x \in \mathbb{R}^{r}$ with $x_{j}>0$ for every $j=1, \ldots, r$. Now the map $\log \left(\lambda_{\min }(a \mid \overline{\mathfrak{n}})\right)$ becomes the map $\lambda(x)=\min _{j} x_{j}, x \in C^{+}$. We will construct $\tau$ as a function on $\mathbb{R}^{r}$. with

Up to scaling our task is to construct a function $\tau \in C_{0}^{m p, m p}\left(\mathbb{R}^{r}, C^{+}\right)$

$$
0<\tau(x)<\left(\min _{j} x_{j}\right)^{p m}, \quad x \in C^{+},
$$

such that $D \tau$ is bounded for every differential operator with constant coefficients of degree $\leq m p$.

Our first approximation to the solution is the function

$$
x \mapsto\left(x_{1} \cdots x_{r}\right)^{m p+1},
$$

which is in $C_{0}^{m p, m p}\left(\mathbb{R}^{r}, C^{+}\right)$, but it satisfies the required estimate only in the set of all $x \in \mathbb{R}^{r}$ with $\|x\|_{\infty}<1$. So we introduce a dampening factor which reduces the growth of the function. We finally define

$$
\tau(x)=c\left(x_{1} \cdots x_{r}\right)^{m p+1} e^{-\left(x_{1}+\cdots+x_{r}\right)},
$$

where $c>0$ is chosen small enough that $\tau$ satisfies the required estimate. The boundedness of $D \tau$ is clear. The lemma is proven.

For $p \in \mathbb{N}$ we finally define the annunciated function $\chi_{p}: A^{-} M \rightarrow[0,1]$ as follows:

$$
\chi_{p}(a m) \stackrel{\text { def }}{=} g_{p}(\tau(a), \sigma(m)) .
$$

Our results yield the following properties of $\chi_{p}$.

- The function $\chi_{p}$ is $p$-times continuously differentiable and invariant under conjugation.

- One has $\chi_{p}(a m) \neq 0 \Leftrightarrow a m \in(A M)^{\sim}$ and $\chi_{p}\left(A^{-} M_{\text {ell }}\right)=1$.

- For every invariant differential operator $D$ on $A$ of degree $\leq k$ one has

$$
D \chi_{p}(\cdot m) C_{0}^{k m p+1+j}\left(A, A^{-}\right) \subset C_{0}^{j}\left(A, A^{-}\right)
$$

for every $j \geq 0$ and every $m \in M$.

From here, one proceeds further as in [1, page $33 \mathrm{ff}$.$] .$ 


\section{References}

[1] A. Deitmar, A higher rank Lefschetz formula. J. Fixed Point Theory Appl. 2 (2007), 1-40.

\section{Anton Deitmar}

Mathematisches Institut

Auf der Morgenstelle 10

72076 Tübingen

Germany

e-mail: deitmar@uni-tuebingen.de 